\title{
Analysis of Multimodal Physiological Signals Within and Between Individuals to Predict Psychological Challenge vs. Threat
}

\author{
Aya Khalaf ${ }^{1}$, Mohsen Nabian ${ }^{2}$, Miaolin Fan $^{3}$, Yu Yin ${ }^{2}$ \\ Jolie Wormwood ${ }^{4}$, Erika Siegel ${ }^{5}$, Karen S. Quigley ${ }^{4}$, Lisa Feldman Barrett ${ }^{4}$, Murat Akcakaya ${ }^{1}$, \\ Chun-An $\mathrm{Chou}^{3}$, and Sarah Ostadabbas ${ }^{2}$
}

\begin{abstract}
Challenge and threat are biopsychological responses following an individual's evaluation of task demands relative to his or her available resources to cope with these demands. In this study, we aimed to investigate individual and group variation in physiological responding across a series of motivated performance tasks of varying difficulty. We specifically tested three hypotheses: (H1) individuals will express different sets of physiological patterns (features) across tasks of varying difficulty; (H2) there will be groups of individuals who share common salient physiological features that dominate within-individual differentiation in physiological responding across tasks of varying difficulty; and $(\mathrm{H} 3)$ the accuracy of predicting self-reported judgments of challenge and threat across individuals will be higher within each group with shared salient physiological features than across all groups or the entire sample. To test these hypotheses, we developed an integrated analytic framework for multimodal physiological data analysis. We employed data from an existing experiment in which participants completed three mental arithmetic tasks of increasing difficulty during which we collected different modalities of physiological data. Analyses revealed three groups of participants who shared common features that best differentiated their within-individual physiological response patterns across tasks. Support vector machine (SVM) classifiers were then trained using both shared features within each group and all computed features to predict challenge vs. threat states. Our results showed that, within-group classification model using person-specific features achieved higher self-report prediction accuracy comparing to the alternative model trained on data from all participants without feature selection.
\end{abstract}

Index Terms-Clustering, Graph Theory, Feature Selection, Multimodal Data Fusion, Support Vector Machine, Mutual Information, Challenge vs. Threat.

\section{INTRODUCTION}

$\mathrm{P}$ SYCHOLOGISTS have documented patterns of cardiovascular physiological response that distinguish challenge and threat states and which occur during motivated performance situations-situations that are goal-relevant to the performer, require instrumental cognitive responses, and are active rather than passive [1]. Challenge and threat responses are related to an individual's perception of situational and task demands (including perceptions of danger, uncertainty, and required effort) relative to one's available resources to cope with these demands (including both dispositional characteristics and external support) [1]-[3]. Challenge is experienced when a person judges his or her coping resources to meet or exceed the demands of the situation or task, whereas threat is experienced when a person judges the situation or task demands to exceed his or her coping resources [1]-[3].Challenge and threat influences important outcomes, including task performance. For ex-

- ${ }^{1}$ Electrical and Computer Engineering Department, University of Pittsburgh, PA, USA.

- 2 Augmented Cognition Lab (ACLab), Electrical and Computer Engineering Department, Northeastern University, MA, USA. Corresponding Author's email: Ostadabbas@ece.neu.edu.

- ${ }^{3}$ Mechanical and Industrial Engineering Department, Northeastern University, MA, USA.

- ${ }^{4}$ Department of Psychology, Northeastern University, MA, USA.

- ${ }^{5}$ Department of Psychology, University of California San Francisco, CA, USA. ample, individuals experiencing threat performed worse on the math portion of the GRE when compared to individuals experiencing challenge [4]. Challenge and threat predict across a wide variety of social contexts including during interpersonal interactions. Individuals are more likely to experience threat when interacting with individuals who are higher in social status [5], are from a racial group other than their own [6]-[8], are socially stigmatized (such as having a visible facial birthmark) [7], or when their interaction partner violates a known stereotype (such speaking with an Asian male with a southern accent) [9].

According to the biopsychosocial model (e.g., [1], [10]), challenge responses are associated with enhanced ventricular contractility (as measured by pre-ejection period or PEP) and increased cardiac output (CO), as well as decreasing systemic vascular resistance (measured as total peripheral resistance or TPR) [1], [11], a pattern of cardiovascular reactivity that should increase blood flow to the periphery to enhance blood flow to skeletal muscles and the heart, thereby supporting greater motor activity. Threat, on the other hand, is associated with increased systemic vascular resistance [1], [11], a pattern of cardiovascular reactivity that reduces efficient and effective blood flow to peripheral blood vessels, and thereby provides poor or limited support of increased muscle action. In the psychophysiological literature [2], [3], [12]-[14], challenge and threat responses have typically been indexed via patterns of task-related change 
in pre-ejection period (PEP), heart rate (HR or the inverse of $\mathrm{HR}$, inter-beat interval or IBI), cardiac output (CO), and/or stroke volume (SV), and total peripheral resistance (TPR).

Thus, experiences of psychological challenge and threat are relevant across many social evaluative and motivated performance situations, predict important outcomes like task performance, cooperative behavior, and interpersonal relationships, and are associated with consistent patterns of cardiovascular reactivity, which can be adaptive or maladaptive for cardiovascular health [8], [15].

In the present study, we intend to extend the existing literature on psychological challenge and threat in several ways. First, although previous research has investigated both within- and between-subject variability in responding during emotional episodes more broadly [16]-[19], these innovations have not yet been leveraged in the literature on challenge and threat responses during motivated performance tasks. Instead, the vast majority of the literature has examined group-level differences in challenge vs. threat responding within a single motivated performance context across stipulated groups (e.g., groups based on subject variables like race or gender, or based on an experimenterdefined condition), whereas few studies have examined individual differences in challenge vs. threat responses across contexts. Thus, we intend to examine individual differences across different versions of a motivated performance task that should vary in difficulty and stressfulness and to integrate across a broader set of multimodal physiological signals to discover whether there are more individually-diverse patterns of cardiovascular response to these motivated performance tasks than have been previously recognized.

Second, we intend to extent the existing literature by examining whether additional physiological variables could distinguish challenge from threat experience. After the initial study demonstrated the basic challenge vs. threat distinction [2], more recent studies focused almost exclusively on cardiovascular responses during motivated performance, without systematically investigating the possible utility of additional indices of peripheral physiological activity for understanding biological responding in active coping stress tasks. However, there are potential advantages to using additional multimodal information so that we can improve prediction accuracy (for example, as in [20]), and potentially also examine whether physiological variables beyond cardiovascular ones, are useful in distinguishing challenge and threat experiences. To address this, in the present study, we included a much broader range of peripheral physiological measures than those typically included in previous studies on challenge and threat, and used them as input to our proposed feature selection and classification algorithms. In addition to indices of cardiovascular responding that are typically investigated within this literature, we also examined measures of facial muscle activity (including activity in the corrugator supercilii and zygomaticus major muscle groups), respiration, and electrodermal activity. A schematic of our study design, data pre-processing and data analysis is shown in Figure 1.

In the present investigation, we first focused on examining within-individual variation in physiological activity corresponding to a series of active coping stressor tasks of increasing difficulty (i.e., with increasing task demands over iterations of the task). We hypothesized that, within individuals, patterns of physiological responding will change as the task demands increase and become more stressful (Hypothesis 1). We next identified person-specific physiological features that best differentiated within-individual physiological responding across tasks of varying difficulty. Although the existing literature suggests that PEP, IBI, CO, and TPR should best distinguish physiological responding across tasks of varying difficulty for all individuals (as individuals likely move from feeling more challenged to more threatened), we hypothesized that there would be groups (clusters) of participants who share common salient features that dominate this differentiation in physiological responding across tasks (Hypothesis 2). We next applied a network analysis approach to characterize these groups and investigated how the shared salient features varied across groups. That is, we predicted that not all participants' stress responses would be best differentiated by the same small subset of cardiovascular variables. Further, by leveraging a data set with a larger set of peripheral physiological variables than are typically used in the challenge and threat literature coupled with an individual difference-focused analytic strategy, we hypothesized that we could reveal heretofore undetected patterns of physiological responding during active coping. Finally, we hypothesized that challenge vs. threat (defined as the ratio of self-reported stress to self-reported coping resources) would be better predicted using the shared salient features within each group versus when using the same set of physiological features for all individuals in the sample as a whole (Hypothesis 3).

\section{Related Work}

The seminal work documenting physiological patterns of challenge and threat was conducted in the 1990s by Tomaka, Blascovich, and colleagues [2], [3]. They demonstrated that the physiological responses to an active coping stressor task differed across individual participants with some having greater cardiac reactivity and less vascular reactivity, whereas other participants had the opposite pattern of responding (i.e., increased vascular constriction and only modestly increased cardiac reactivity). These patterns of physiological responding were significantly related to participants' self-reports of the expected stressfulness of the task and expected ability to cope with the task - a priori judgments of being adequately resourced to cope with the stress of the upcoming task (i.e., challenge appraisals) were associated with decreased vascular resistance and increased blood flow to the periphery, measured as cardiac output. Conversely, a priori judgments of being inadequately resourced to cope with the stress of the upcoming task (i.e., threat appraisals) were associated with increased vascular constriction and lesser cardiac reactivity [2]. Moreover, the researchers argued for a causal relationship between stress/coping appraisals and patterns of physiological responding, as manipulations of peripheral physiology did not produce corresponding changes in self-reported experiences of stress and coping, whereas those experiences predicted physiological responding [3].

In the decades since their scientific introduction, challenge and threat have been used to understand psycholog- 
I. Experiment \& Data Collection

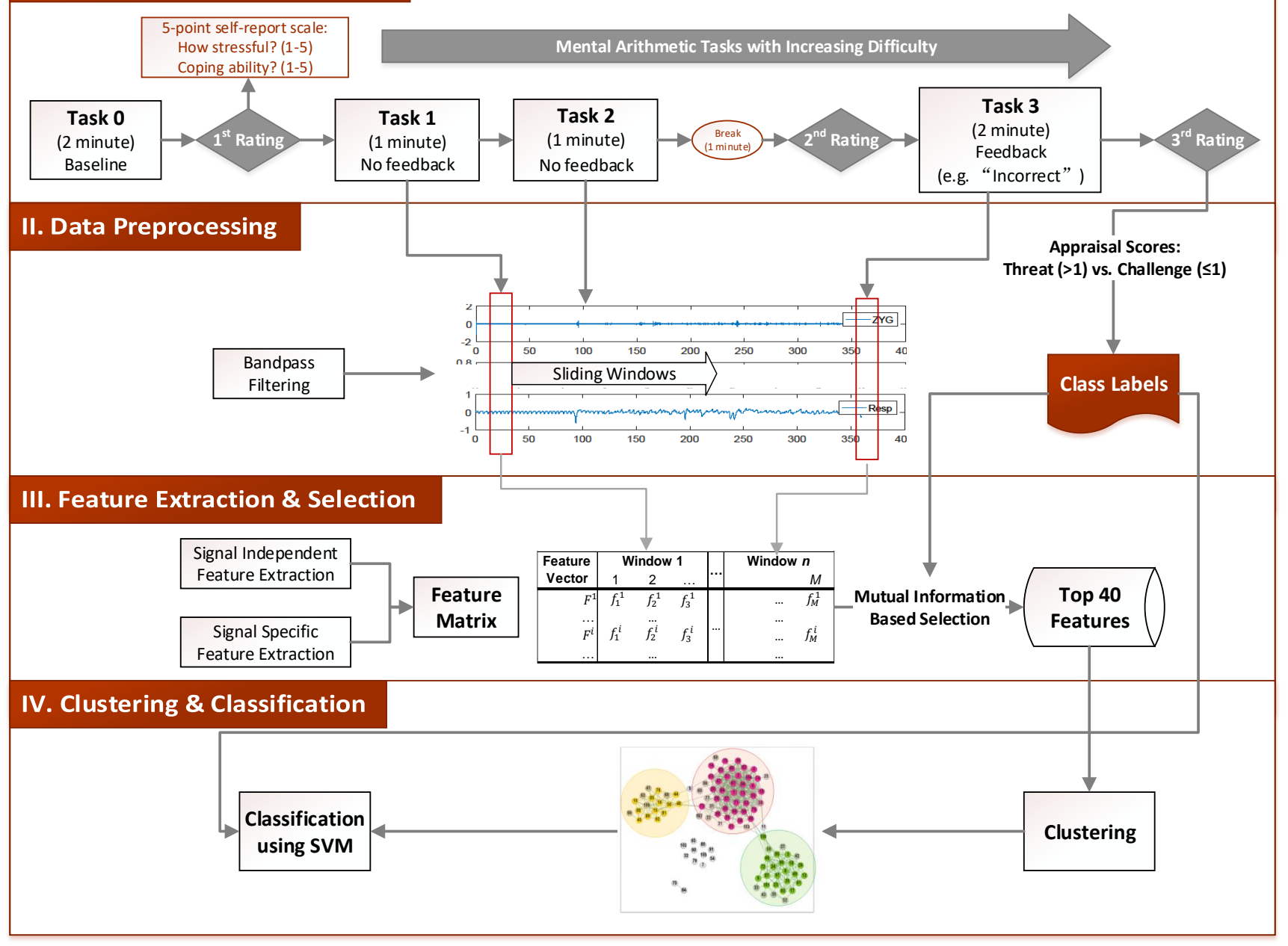

Fig. 1. The methodological framework of this study. I. In the experiment phase, four mental arithmetic tasks with increasing difficulty were conducted to collect multimodal physiological data as well as self-report scores from participants. II. Following a band-pass filter and normalization, sliding windows (ten seconds length for EDA and five seconds length for other modalities) with $75 \%$ overlap were used for segmenting the signals. III. Two types of feature extraction approaches were applied to the segmented data and obtained the feature matrix, where each row represented a feature vector of one participant. Then, we selected top 40 features based on the mutual information between feature vectors and class labels (converted from self-reported scores). IV. A distance-based clustering algorithm was performed based on the selected subset of features, and SVM classifier was trained for within- and across group classification.

ical, behavioral, and biological responses across many motivated performance domains, particularly those involving social evaluation [5]-[9]. However, the vast majority of this work has not examined challenge and threat responses at the individual level, focusing instead on group comparisons between samples of individuals who either exhibit more threat-like versus more challenge-like experiences or more threat-like or challenge-like changes in physiology. A renewed focus on within-individual comparisons hinders the identification of potentially important individual differences in challenge and threat response patterns that could better predict experience and behavior across different motivated performance contexts. This would be the first step to improving the prediction of stress-related health related outcomes that the biopsychosocial model was originally designed for and that are sorely needed. In one exception to this group-based approach, Quigley, Barrett, and col- leagues investigated individual patterns of cardiovascular responding across a series of motivated performance tasks of increasing difficulty [14]. In this experiment, participants completed four mental arithmetic tasks, which required them to perform serial subtractions aloud (e.g., "subtract $7 \mathrm{~s}$ from the number 3,746") in the presence of an evaluator. Each iteration of the task increased in difficulty by adding new elements of social evaluative pressure as the experiment progressed. The experience of threat and challenge (measured as ratings of stress and coping resources for each task) were assessed before each task and cardiovascular physiological reactivity was measured before, during and after every task. However, from an idiographic perspective, researchers also indicated that the relationship between the self-reported judgments and cardiovascular reactivity may be altered due to the willingness to report true level of threats, influenced by the participants' individual-specific 
variables.

These findings demonstrated the importance of examining individual-specific patterns of challenge and threat responses idiographically as opposed to at the group-level. Thus, we hypothesize that (H1) individuals will express different sets of physiological patterns (features) across active coping stressor tasks of varying difficulty; (H2) there will be groups of individuals who share common salient physiological features that dominate within-individual differentiation in physiological responding across tasks of varying difficulty; and (H3) the accuracy of predicting self-reported cognitive judgment ratings across individuals will be higher within each group with shared salient physiological features than across all groups or the entire sample.

\section{Materials and Methods}

\subsection{Participants}

260 participants were recruited from the greater Boston area to participate in this experiment. Here, we used data from the 107 participants (41 males, 65 females; 1 gender not reported) who had complete, clean physiological data for all tasks. The remaining 152 participants had data from at least one physiological measure for at least one minute of either the tasks or baselines that was not usable due to excessive movement artifact. Participants were required to be native English speakers and were excluded if: they were currently taking any medications that would influence peripheral physiological activity, had a history of mental illness or cardiovascular disease, or reported consuming caffeine, alcohol, or tobacco within 24 hours prior to the experiment. All participants completed the mental arithmetic tasks as part of a single 3-4 hour experimental session in which they completed a series of other tasks and questionnaires not relevant to the current investigation. The study was approved by the Institutional Review Board at Northeastern University.

\subsection{Measures}

\subsubsection{Physiological Responding Signals}

We recorded the electrocardiogram (ECG), the impedance cardiogram (ICG), continuous blood pressure (BP), respiration (RES), electrodermal activity (EDA), and measures of facial muscle activity over the corrugator supercilii (COR) and zygomaticus (ZYG) major muscle groups using facial electromyography (fEMG). All physiological measures were sampled at $1000 \mathrm{~Hz}$ using BioLab v. 3.0.13 (Mindware Technologies; Gahanna, $\mathrm{OH}$ ) via a BioNex 8-Slot chassis (Model 50-3711-08). Samples of raw, filtered physiological data from one participant are shown in Figure 2. The filter settings for each bio-signal is later discussed in the Data Preprocessing section.

ECG was obtained using pre-gelled $\mathrm{Ag} / \mathrm{AgCl}$ sensors in a modified lead II configuration. ICG was acquired using a four-spot electrode configuration (see [21]) using pre-gelled $\mathrm{Ag} / \mathrm{AgCl}$ electrodes. The inner (recording) electrodes were placed on the participant's chest: one at the base of the neck at the top of the sternum and the other at the level of the xiphisternal junction. The outer (source) electrodes were

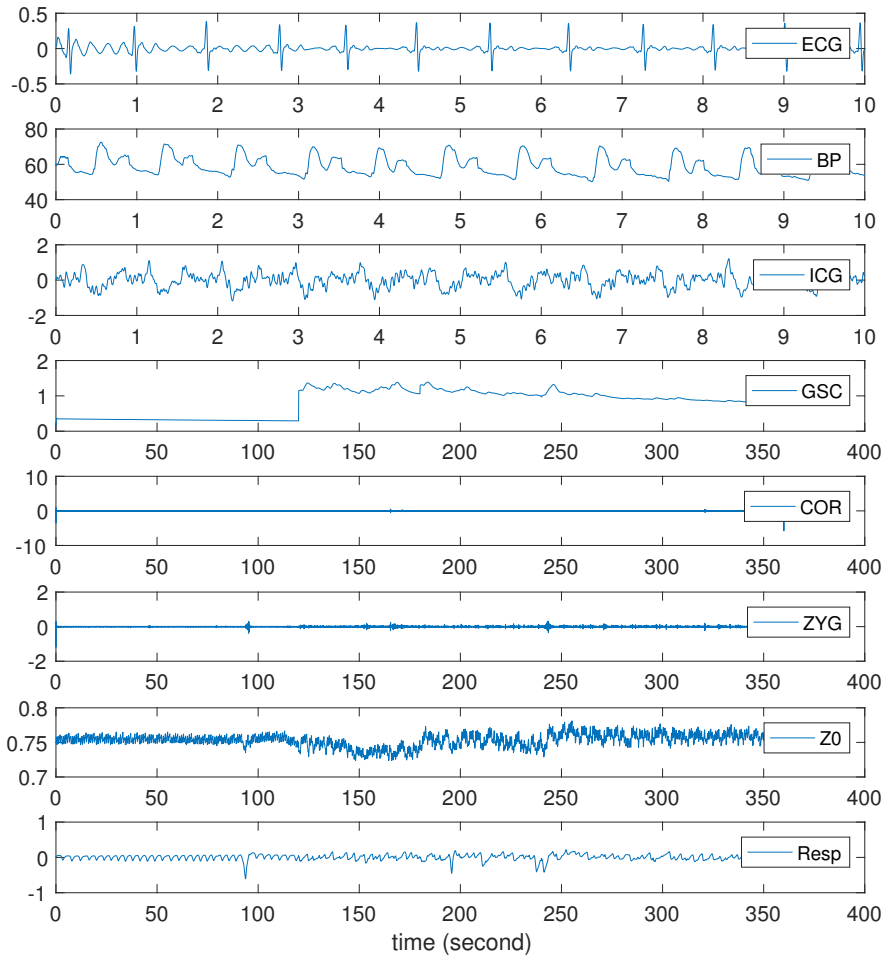

Fig. 2. Samples of collected physiological signals after preprocessing. The first three signals, ECG, BP and ICG contain high frequency components and only the first 10 seconds of these three signals are showing here. The rest of the signals with lower frequency components are shown during the whole data length ( $=6$ minutes).

placed along the midline on the participant's back approximately $4 \mathrm{~cm}$ above and below the inner recording electrodes (respectively, roughly over the fourth cervical vertebrae and the ninth thoracic vertebrae). The source electrodes passed a $4 \mathrm{~mA}, 100 \mathrm{kHz}$ alternating current across the thorax. BP was recorded continuously via a Continuous Noninvasive Arterial Pressure monitor (CNAP Monitor 500AT; CNSystems; Medizintechnik, AG, Austria). Continuous recordings were obtained from small cuffs placed on participants' left middle and pointer fingers, and these continuous readings were calibrated intermittently against non-invasive, blood pressure measurements taken from a cuff around the participant's right upper arm. The RES signal was measured via a piezoelectric belt placed around the lower-chest/upperabdomen (Mindware Technologies; Model 50-4504-00). The EDA signal was recorded from the right palmar surface of the hand with sensors on the thenar and hypothenar eminences using disposable, $\mathrm{Ag} / \mathrm{AgCl}$ (11mm diameter; isotonic paste) electrodes (Biopac Systems, Inc.; Goleta, CA). fEMG measures were obtained via reusable $\mathrm{Ag} / \mathrm{AgCl}$ electrodes (Mindware Technologies; Gahanna, $\mathrm{OH}$ ) filled with an electrolyte gel and placed over the zygomaticus major and corrugator supercilii muscle regions on the right side of the participant's face. A reference electrode was placed in the middle of the forehead.

\subsubsection{Procedure: Mental Arithmetic Tasks}

Each participant completed a series of mental arithmetic tasks as part of a single experimental session, which typically lasted between 3 and 4 hours, during which s/he 
completed a number of other tasks unrelated to the current investigation. Following electrode placement, the participant was seated in a sound-attenuated testing room in an upholstered chair. Immediately before completing the series of mental math tasks pertinent to the current investigation, the participant was connected to the continuous blood pressure monitor, and the continuous readings from the finger cuffs on the left hand were calibrated against a non-invasive reading taken from the cuff on the right arm. The participant then sat quietly alone in the testing room while resting physiological measures were recorded for 2 minutes (Task 0 or Baseline). The participant was not informed that $\mathrm{s} / \mathrm{he}$ would be doing arithmetic tasks until after the baseline to ensure that this resting baseline was not confounded with anticipatory stress related to the upcoming tasks.

After the baseline, the participant was informed that $\mathrm{s} /$ he would complete a series of mental arithmetic tasks by speaking serial subtraction answers aloud in front of an experimenter, who would recording the answers. The participant was asked to work as quickly and accurately as possible, and to refrain from commenting on the task or his or her performance until each task was compete. The participant then practiced by subtracting aloud from 90 by 3s for 30 seconds, and was encouraged to ask any questions about the task instructions. Next, the participant rated how stressful the upcoming task would be and how well s/he could cope with the upcoming task on two 5-point scales (from $1=$ Not at All to $5=$ Very Much). Immediately following these ratings, the first subtraction task began and the participant was asked to subtract from 725 by $7 \mathrm{~s}$ for 1 minute while an experimenter recorded the responses (Task 1). Experimenters were trained not to provide any positive feedback (e.g., no smiling or nodding) during the task. For the second arithmetic task, the participant again subtracted aloud for 1 minute, this time from 847 by $6 s, 8 s$, or $13 \mathrm{~s}$ with the goal of keeping the serial subtractions moderately stressful for all participants and adjusting the task difficulty to their initial performance (Task 2). Again, the experimenter recorded his or her responses, but gave no feedback.

The participant next was given a short ( 1 minute) break to sit quietly alone in the testing room, after which the experimenter returned to the room, and the participant was told that there would be one last math task, but that this time $\mathrm{s} /$ he would subtract from a larger number and that the experimenter would inform the participant each time an incorrect response was given. These changes were meant to increase the stressfulness of the serial subtractions in the final two-minute task. Before these subtractions, the participant again rated how stressful s/he thought the upcoming task would be and how well s/he could cope with the upcoming task on the same 5-point scales. The participant then was asked to subtract from 4851 by $8 \mathrm{~s}, 12 \mathrm{~s}$, or $17 \mathrm{~s}$ for two minutes (again depending on performance on the first minute of the task) (Task 3). For these two minutes, the experimenter recorded the participant's answers and provided feedback each time s/he was incorrect (e.g., "Incorrect. 4826"). Immediately following this final two minute task, the participant rated how stressful the task was and how well s/ he coped during the task using the same 5-point scales. The ratio of the two ratings (stress rating/coping rating) is defined as the participant's self-reported experience of threat or challenge $(S R)$. Ratios greater than " 1 " indicate a threat experience, while self-report ratings of less than or equal to " 1 " indicate a challenge experience.

\subsection{Hypothesis-Driven Models}

\subsubsection{Problem Formulation to Test $\mathrm{H} 1$}

With the aim of finding the physiological features that best differentiate a given individual's pattern of bodily response across tasks of varying difficulty/stress, a classifier will be trained to differentiate 4 classes $\left(T_{0}=\right.$ baseline, $T_{1}=$ Task $1, T_{2}=$ Task 2, $T_{3}=$ Task 3 ) for each participant. These features are also believed to be the most experienceindicative features.For a given individual, $i$, let's extract $M$ features from the available physiological signals collectively to form feature vector $\mathcal{F}^{i}=\left[f_{1}^{i}, f_{2}^{i}, \ldots, f_{M}^{i}\right]^{T}$ in each class of $\left\{T_{0}, T_{1}, T_{2}, T_{3}\right\}$. The objective is to find a subset of $K_{i}$ feature from $\mathcal{F}^{i}$ in which the classification accuracy when differentiating these 4 classes is more than a predefined accuracy threshold, $\mathcal{T}$.

\subsubsection{Problem Formulation to Test $\mathrm{H} 2$}

To test this hypothesis, the objective is to find $J$ clusters (groups) of individuals, $\left\{C_{1}, \cdots, C_{J}\right\}$ that share a common subset of salient features in differentiating $\left\{T_{0}, T_{1}, T_{2}, T_{3}\right\}$ classes, with a predefined commonality measure greater than $\mathcal{L}$.

\subsubsection{Problem Formulation to Test $\mathrm{H} 3$}

To test this hypothesis, an inference model, $\mathcal{I}$ is defined to predict self-report ratings reported at the end of Task 3 using the feature vector extracted from class $T_{3}$. The self-report rating $S R$ is predicted in a binary fashion: $S R>1$ for threat and $S R \leq 1$ for challenge. To predict the self-report ratings $S R$ of the participants in the cluster $C_{j}, j \in[1, \ldots, J]$, we will train $\mathcal{I}$ in the following structure and test it in a leaveone-out paradigm:

(i) training $\mathcal{I}_{i}$ on the union of the $K_{i}$ feature subset of each participant in the cluster $C_{j}$ and testing it across the participants in the cluster $C_{j}$.

(ii) training $\mathcal{I}_{i i}$ on the union of the $K_{i}$ feature subset of each participant in the cluster $C_{j}$ and testing it with the participants outside the cluster $C_{j}$.

(iii) training $\mathcal{I}_{i i i}$ on the union of the $K_{i}$ feature subset of each participant outside the cluster $C_{j}$ and testing it with the participants in the cluster $C_{j}$.

(iv) training $\mathcal{I}_{i v}$ with all the features on all participants of $C_{k}$, where $k \in[1, \ldots, J]$ and testing the model with the participants from cluster $C_{j}$.

and (v) training $\mathcal{I}_{v}$ on all feature set $\mathcal{F}$ and across all of the $N$ test participants, without any clustering and testing it with all of the participants.

\subsection{Physiological Data Preprocessing}

Physiological signals including Electrocardiogram (ECG), Electrodermal Activity (EDA) and Electromyogram (EMG) were processed using the bio-signal processing MATLAB toolbox developed at Augmented Cognition Lab (ACLab) at Northeastern University [22]. The toolbox extracts features on these signals based on the state-of-the-art studies reported in scientific literature [23]-[28]. Processing of 
other signals such as Blood Pressure (BP), Respiration (RES) and Impedance Cardiography (ICG) were also performed in MATLAB based on the recommendations suggested in scientific articles [2], [3], [29], [30]. In this section, the preprocessing steps are explained and, in the following sections, the data segmentation and feature extraction of the signals are elaborated.

In the pre-processing of the ECG signals, an Elliptic band-pass filter with cut-off frequencies of $5-45 \mathrm{~Hz}$ was used. These cut-off frequency values were selected based on the power spectral density (PSD) analysis of the ECG signals and the Elliptic filter was selected to ensure the amplitude of the peak points on the signal were not significantly suppressed by the filter [31].

For the EDA signals, FIR low-pass filter with $0.5 \mathrm{~Hz}$ cutoff frequency was used [32].An elliptic bandpass filter with cutoff frequencies of $10-300 \mathrm{~Hz}$ was used for both the COR and ZYG signals recorded via fEMG. To reduce the baseline noise and artifact noise, and also preserve the desired information from sEMG signal, a band-pass filter was chosen to compromise. [33] By the power spectral density inspection of the EMG signals, an Elliptic filter with the cutoff frequency of $10-300 \mathrm{~Hz}$ was selected as the optimal choice for the EMG filter. Moreover, according to the power spectrum of the two EMG signals acquired for each participant, two notch filters at $60 \mathrm{~Hz}$ and $180 \mathrm{~Hz}$ were used.

The recommended low pass cut-off frequency of the BP signals for the human is in the range of $100 \mathrm{~Hz}-200 \mathrm{~Hz}$ [29]. After the power spectral density analysis of the BP signals, it was concluded that cut-off frequency of $100 \mathrm{~Hz}$ performs optimally. As for the respiration signal and transthoracic basal impedance signals (Z0), we used the Butterworth low pass filter with a cut-off frequency of $20 \mathrm{~Hz}$.

Finally, for the ICG, a 2nd order Butterworth bandpass filter with a cut-off frequency of $0.75-40 \mathrm{~Hz}$ was used based on the recommendation provided in BioLab software [34].

\subsection{Physiological Data Segmentation}

All physiological signals were subjected to the same segmentation process. Data segments corresponding to the baseline $\left(T_{0}\right)$ as well as to Task $1\left(T_{1}\right)$, Task $2\left(T_{2}\right)$ and Task $3\left(T_{3}\right)$ were obtained using time stamps for the start and end of each segment provided in a separate text file for each participant. In the within-individual classification problems, in order to extract features from the segment corresponding to each task, a 5-second window that slides over each task segment was employed with 50 percent overlap for 7 out of the recorded 8 signals. Since the EDA is a slowly changing signal, a sliding window of 10 seconds duration and 50 percent overlap was considered.Each window was considered as an observation belonging to a specific task and used for feature extraction.The same window size and overlap were used for the between-subject classification problem. To reduce variability due to the differences in signal range from person to person in the between-subject problem, the features extracted from each baseline window were subtracted from the features of the corresponding $T_{3}$ window.

\subsection{Feature Extraction}

Several sets of features were extracted from the 8 recorded physiological signals and employed in a machine learning framework to initially differentiate patterns of physiological responding across tasks of varying difficulty levels. The goal is to find the set of features that is most related to changes in bodily responding across various task demands, as such set of features should correspond with changes in challenge vs. threat experiences across tasks. The features included signal-independent and signal-specific features. Signal-independent features included Fourier based features while signal-specific features included features designed especially for extracting information from the ECG, EMG, EDA, BP, and ICG signals.

\subsubsection{Signal-Independent Features}

The power spectrum was estimated for each window of data using Welch method [35]. The raw power spectrum values were considered as features. For each window per signal, the number of features obtained from the power spectrum was reduced by calculating the average power over a narrow range of frequencies instead of using all the values as features. The average of power spectrum values within a sliding window of specific width was considered as one feature. To calculate the same feature for the next window, the original window is shifted by a value equal to its width so that there is no overlap between consecutive windows and the average of that window is calculated and so on. For ECG, respiration, Z, and ICG signals, the average power was calculated using a window of $2.5 \mathrm{~Hz}$ width. Since the blood pressure signal has higher bandwidth compared to the previously stated signals, and considering the need to reduce the number of features, a window of $5 \mathrm{~Hz}$ width was used. For the same reason, a window of $10 \mathrm{~Hz}$ width was employed for the EMG signals.

\subsubsection{Signal-Specific Features}

ECG features: ECG records electrical activity of the heart that contains rich information about heart functionality, sleep quality and affective experience [24], [36]. In this paper, within each window, we calculated mean R-R intervals (i.e., the time between consecutive heartbeats), standard deviation of R-R intervals, standard deviation of the differences between adjacent R-R intervals and the square root of the mean of the sum of the squares of differences between adjacent R-R intervals. Another set of calculated features included the number of pairs of adjacent $R-R$ intervals where the first $R-R$ interval exceeds the second $R-R$ interval by more than $50 \mathrm{~ms}$ as well as the number of pairs of adjacent $R-R$ intervals where the second $R-R$ interval exceeds the first R-R interval by more than $50 \mathrm{~ms}$. Mean area of each QRS complex is also calculated in addition to its standard deviation.

EMG features: EMG records the electrical activity produced by skeletal muscles. The calculated features include standard deviation, root mean square, and mean absolute value of the EMG signal within each window. In addition, features related to the signal frequency were calculated, including the number of zero crossings and the number of times the slope sign changed. Another feature is the 
cumulative length of the EMG signal within the analysis window, which provides a measure of the complexity of the signal. In addition, an estimate of the exerted muscle force (f) for a signal $\mathbf{x}$ of $\mathrm{N}$ samples is calculated as:

$$
f=e^{1 / N \sum_{k=1}^{k=N} \log x_{k}}
$$

EDA features: Electrodermal activity (EDA) measures changes in the skin's electrical conductivity due to changes in the amount of sweat present in the eccrine sweat glands of the palm. The EDA signal is composed of two activities, tonic activity represented by the slowly varying base signal and phasic activity or skin conductance responses (SCRs) which are represented by faster variations in the signal. SCRs was detected by performing differentiation and subsequent convolution with a 20-point Bartlett window. In this paper, we extracted signal mean, number of detected SCRs, mean SCR duration, mean SCR amplitude, and mean SCR rise-time (where rise-time of an SCR is defined as the time between the initial rise and the peak of an SCR).

BP features: The characteristic peaks for the BP signal were used for calculating signal features for BP. These peaks included systolic, diastolic, dicrotic and notch peaks. Based on the extracted peaks, we calculated 5 features. The first one is the diastolic pressure which is the minimum signal value for each beat. The second one is the systolic pressure defined as the maximum signal value for each beat. Pressure values at both the dicrotic and notch peaks were also calculated. Mean arterial pressure (MAP), which represents mean signal value for each beat, was also considered as a feature.

ICG features: Features calculated from the Impedance Cardiogram included left ventricular ejection time (LVET), stroke volume (SV), cardiac output (CO), pre-ejection period (PEP) and total peripheral resistance (TPR). LVET is the time period in which blood flows across the aortic valve, and PEP is the time period between the electrical activity signaling the start of ventricular contraction and the onset of blood being ejecting into the Aorta [37]. SV is defined as the blood volume pumped by the heart per heart beat, and $\mathrm{CO}$ is the blood volume pumped by the heart per minute [38]. Finally, TPR represents the resistance of the systemic circulation that carries oxygenated blood from the heart to the body, and returns deoxygenated blood to the heart. These five variables were extracted from the shape characteristics of ICG signal combined with the corresponding ECG signal,Z0 signal and BP signal. The methods used to extract these features are based upon the discussions provided in the scientific literature [30], [39].

\subsection{Feature Selection}

In general, the objectives of feature selection are three-fold: improving the prediction performance of the predictors, providing faster and more cost-effective predictors, and providing a better understanding of the underlying process that generated the data. In our work, feature selection is related to the within-individuals classification of bodily responses across the 4 tasks of varying difficulty. Feature selection methods include filter, wrapper, and embedded methods. Compared to wrapper and embedded methods, the advantage of using filter methods is the low computational complexity [40]. However, filter methods assume that the features are independent. Therefore, it is possible that these methods select redundant features. Due to the high dimensionality of the feature vector (159 features), we applied a filter method for feature selection. Filter methods include statistical hypothesis tests such as student-t test and Wilcoxon Rank Sum test, however, these tests were not used in this study since they can work only with binary classification problems. In this work, we decided to use mutual information, which is a filter method, to select the most significant $K_{i}$ features out of the original feature vector $\mathcal{F}^{i}=\left[f_{1}^{i}, f_{2}^{i}, \cdot, f_{M}^{i}\right]^{T}$ for participant $i$. Mutual information measures the contribution of each feature to make a correct decision and assigns each feature a score based on that contribution. In other words, the higher the score is, the higher the contribution is of that feature to correct classification. In this paper, the scores were normalized by the maximum score which corresponds to the most significant feature.

\subsection{Task and Self-Report Rating Classification}

In this paper, for both within-individual task level classification, and across-groups and across-individuals self-report rating classification, we apply a support vector machine (SVM) classifier with a linear kernel function to reduce computational expenses. The goal of SVM herein is to find an optimal hyperplane that discriminates individuals in terms of self-report ratings $(S R>1$ versus $S R \leq 1)$ in the selected feature space. The classification/prediction accuracy is defined as the percentage of individuals correctly classified in the class they supposedly belong to.

\section{Results}

\subsection{Testing Hypothesis 1: A Within individual Analysis}

For each participant, the accuracy of predicting the task was calculated at different numbers of features through a feature selection method. A four-class classification problem has

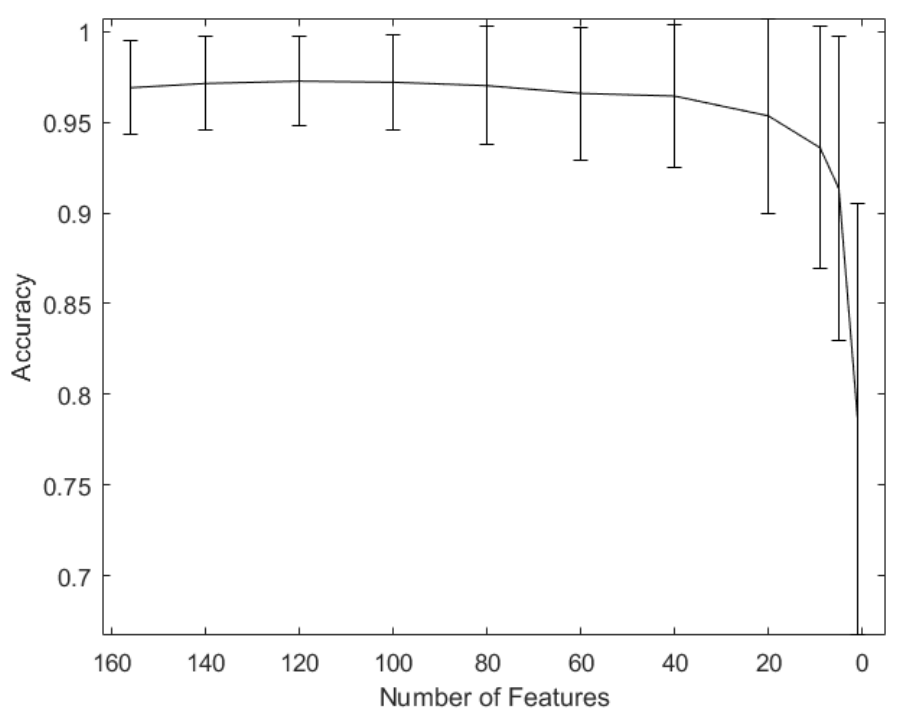

Fig. 3. The average accuracy obtained from 107 subjects plotted at different numbers of selected features. 
been formulated to distinguish among Baseline, Task 1, Task 2 , and Task 3. Due to the limited number of samples per task, a leave-one-out cross validation procedure was employed. In this classification problem, mutual information was used to select the most relevant $\{1,5,10,20, \ldots, 159\}$ features and the corresponding accuracy was calculated. Figure 3 shows the average accuracy obtained over 107 subjects at different numbers of selected features. It is clear that using at least 40 features results approximately in the same accuracy obtained using the whole feature set. Therefore, for each participant, out of 159 possible features, $K_{i}=40$ features are labelled as most significant. This corresponds to features with normalized mutual information scores higher than 0.7 , which also corresponds to a classification accuracy threshold of $\mathcal{T}=95 \%$.

To test our hypothesis $\mathrm{H} 1$, we rely on the histogram of the top 40 subject-specific features across individuals. The results are illustrated in Figure 4. This figure shows that among 149 features, only approximately 20 features appeared in the top 40 features across the majority of the individuals. Also, note that since the histogram is not uniform, we can conclude that some features appear in the top 40 feature list more frequently than the others. This also means that different individuals have different significant features in their top 40 list.

\subsection{Testing Hypothesis 2: Between Subject Clustering Analysis}

We hypothesize that there are subgroups of people who may share common subset of features in their top $K_{i}=40$ selected features. Based on this shared commonality, we suggest that individuals would form subgroups. Moreover, some individuals may not belong to any group. Finding these subgroups is a clustering problem. However, since we do not know the number of the clusters a priori, and also since we do not want to force every individual to be a part of a subgroup/cluster, to solve this problem, we take a graph theoretic approach.

We form a binary feature vector of size $159 \times 1$ (the same size as $\mathcal{F}$ ) and for each individual, if the $i^{\text {th }}$ feature is in their top 40 features, we place 1 in the $i^{\text {th }}$ location of this binary feature vector. Otherwise, a 0 is placed in that location. To cluster the subjects based on the similarity in the binary feature vectors, a graph was constructed by considering each participant as a node and the Jaccard coefficient [41] as the connectivity measure between the binary vectors. A connection is decided to exist between two nodes if the normalized Jaccard coefficient between these nodes is above the predefined commonality measure $\mathcal{C}=0.5$.

Figure 5 shows the clustering patterns of participants in a graph, where three major clusters can be visually observed. The nodes in grey represent individuals that do not belong to any clusters. On the other hand, some of the individuals may have very few connections to one cluster, or some may have connections to multiple clusters. In order to resolve these issues, we computed the cumulative distribution function (CDF) for node degrees of the graph, see Figure 6. Degree for each node is defined as the number of connections that that node makes with the other nodes in the graph. Based on the degree CDF, we identify a threshold, $\rho$ such that (1) if a node has connections only to one cluster with the connectivity degree of $d$, and if $d>\rho$, we determined this node to belong to that cluster; (2) if a node has connections to more than one cluster (i.e., $J$ clusters); assuming that the degree of connectivity to different clusters are defined as $d_{1}$, $d_{2}, \ldots, d_{J}$, if $\max \left(d_{1}, d_{2}, \ldots, d_{J}\right)>\rho$, then that belongs to the cluster with the maximum connectivity degree. In our results, we selected $\rho=5$ which corresponds to degree CDF higher than 0.7.

By considering the most frequent significant features for the participants within each cluster, shown in Table 1, where Morph represents morphological features, we found that the small cluster containing 15 participants is dominated by the power spectrum features from COR signal while the second cluster containing 22 participants is dominated by the power spectrum features derived from the Z0 signal. Moreover, the third cluster with 37 participants is dominated by the ZYG power spectrum features. Such analysis reveals that the top stress -indicative physiological signals shared among the majority of the individuals participated in this study are those recorded from facial muscles. As for the rest of individuals, the transthoracic basal impedance is shown to be the top common stress-related signal.

\subsection{Testing Hypothesis 3: A Generalization Analysis}

As described in Section 3.3.2, and based on the subgroups/clusters obtained in Section 4.2, self-report, we performed a classification/prediction analysis using the selfreport ratings obtained at the end of Task 3 to test our hypothesis that experiences of threat and challenge would be better predicted within each subgroup than across the entire group of participants. The results are presented in Table 2. In this table, for each cluster the prediction accuracy and prediction sensitivity results are presented for 4 different inference models, $\mathcal{I}_{i}, \mathcal{I}_{i i}, \mathcal{I}_{i i i}$, and $\mathcal{I}_{i v}$. Here, (a) $\mathcal{I}_{i}$ is the model used to obtain within-cluster prediction results; (b) $\mathcal{I}_{i i}$ is the model used to analyze the generalization of the prediction accuracy from one cluster to outside that cluster; and (c) the models $\mathcal{I}_{i i i}$ and $\mathcal{I}_{i v}$ are trained to investigate the generalization of the prediction accuracy from outside one cluster to inside that cluster. Moreover, in Table 2, the model $\mathcal{I}_{v}$ evaluates the self-report rating prediction accuracy over the entire group without considering subgroup characteristics.

In Table 2, we observe that the best self-report rating prediction accuracy has been achieved when the prediction is performed within each specific subgroup/cluster with participants sharing common salient features (inference model $\mathcal{I}_{i}$ ). We also observe that when the inference model is trained within a subgroup and applied to participants outside that subgroup (inference model $\mathcal{I}_{i i}$ ), the prediction accuracy of that model outside the subgroup compared to the within-subgroup accuracy has significantly dropped. Moreover, for a specific subgroup sharing common features, when an inference model is trained using these shared features obtained from the participants outside this subgroup and applied to the participants inside the subgroup (inference models $\mathcal{I}_{i i i}$ and $\mathcal{I}_{i v}$ ), the generalization of this inference model from outside to within the subgroup was very poor compared to within subgroup prediction. Finally, 


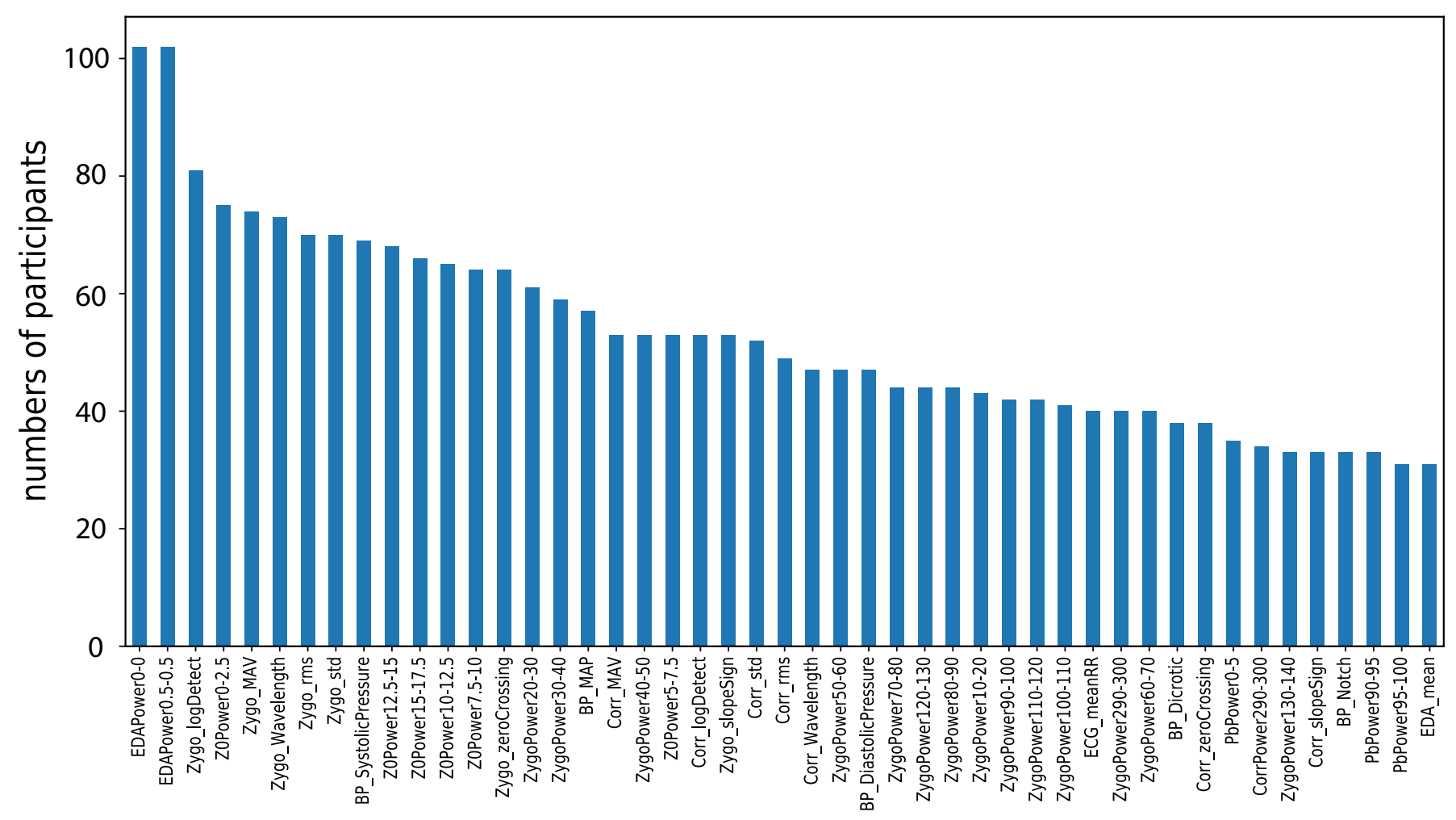

Fig. 4. Distribution of the features appearing among the top $K_{i}=40$ features across all individuals. (only features appeared in at least one-third of all individuals are shown, 47 features out of total 159 features). Features associated with numbers are the power spectrum features. Numbers existing beside these features reflect the frequency range over which this feature is calculated.

TABLE 1

The top common significant feature sets across the 3 clusters and the isolated participants. The most common feature set across a group is represented by " 1 " and the number increases as the feature set commonality decreases.

\begin{tabular}{|c|c|c|c|c|c|c|c|c|c|}
\hline Significant Features & Corr Power & Corr Morph & Zygo Power & Zygo Morph & Z0 Power & EDA Power & BP Power & BP Morph & ECG Morph \\
\hline C1 (15 participants) & 1 & 4 & - & - & 2 & 3 & - & 5 & - \\
\hline C2 (22 participants) & - & - & - & 4 & 1 & 2 & 3 & 6 & 5 \\
\hline C3 (37 participants) & - & - & 1 & 4 & 2 & 3 & - & 5 & - \\
\hline Others & - & 3 & - & 4 & 1 & 2 & - & 5 & - \\
\hline
\end{tabular}

TABLE 2

The accuracy of different inference models $\mathcal{I}$ 's to predict self-report rating $S R$ for 3 clusters.

\begin{tabular}{l|c|c|c}
$\begin{array}{l}\text { Inference } \\
\text { Models }\end{array}$ & $\begin{array}{c}\text { Sensitivity } \\
(S R \leq 1)\end{array}$ & $\begin{array}{c}\text { Sensitivity } \\
(S R>1)\end{array}$ & $\begin{array}{c}\text { Prediction } \\
\text { Accuracy }\end{array}$ \\
\hline$C 1: \mathcal{I}_{i}$ & $76.33 \%$ & $74.47 \%$ & $75.46 \%$ \\
$C 1: \mathcal{I}_{i i}$ & $61.70 \%$ & $35.70 \%$ & $50.24 \%$ \\
$C 1: \mathcal{I}_{i i i}$ & $87.50 \%$ & $0.00 \%$ & $46.67 \%$ \\
$C 1: \mathcal{I}_{i v}$ & $80.05 \%$ & $0.30 \%$ & $42.84 \%$ \\
\hline$C 2: \mathcal{I}_{i}$ & $88.01 \%$ & $71.18 \%$ & $79.59 \%$ \\
$C 2: \mathcal{I}_{i i}$ & $64.52 \%$ & $28.52 \%$ & $49.04 \%$ \\
$C 2: \mathcal{I}_{i i i}$ & $80.27 \%$ & $14.31 \%$ & $47.29 \%$ \\
$C 2: \mathcal{I}_{i v}$ & $91.48 \%$ & $08.89 \%$ & $50.19 \%$ \\
\hline$C 3: \mathcal{I}_{i}$ & $69.15 \%$ & $49.06 \%$ & $60.00 \%$ \\
$C 3: \mathcal{I}_{i i}$ & $49.15 \%$ & $43.99 \%$ & $46.90 \%$ \\
$C 3: \mathcal{I}_{i i i}$ & $67.45 \%$ & $43.00 \%$ & $56.30 \%$ \\
$C 3: \mathcal{I}_{i v}$ & $100.00 \%$ & $02.88 \%$ & $55.38 \%$ \\
\hline$A L L: \mathcal{I}_{v}$ & $84.79 \%$ & $14.45 \%$ & $53.53 \%$
\end{tabular}

when an inference model is trained within the entire population using the union of features shared by this population and applied to the entire population (inference model $\mathcal{I}_{v}$ ), the self-report rating prediction accuracy is lower than the within subgroup prediction accuracy. Please also note that when the generalization of the prediction from within the subgroup to outside the subgroup or from outside the subgroup to within the subgroup or across the entire population is considered, the inference models were able to identify the experiences of challenge very accurately $S R \leq 1$; i.e., sensitivity of predicting $S R \leq 1$ was very high. However, these inference models were less successful in predicting threat experiences $(S R>1)$. Therefore, the overall accuracy was lower compared to within subgroup prediction accuracy. We believe that the reason for this outcome is that for low self-report ratings the features are shared across the entire population. On the other hand, the features that determine the high self-report ratings vary significantly across subgroups; and hence, these features determine the separation of subgroups within the entire population. 


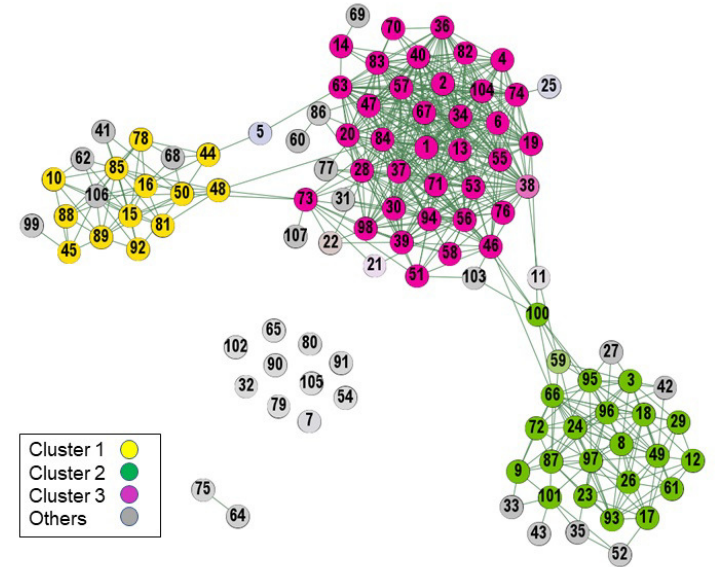

Fig. 5. The clustering structure of participants is represented in this graph by coloring. Each node represents a participant, and the connectivity is constructed based on commonality in the top 40 salient physiological features. Three major clusters are shown in yellow, green, and red, while the remaining nodes in gray represent individual clusters.

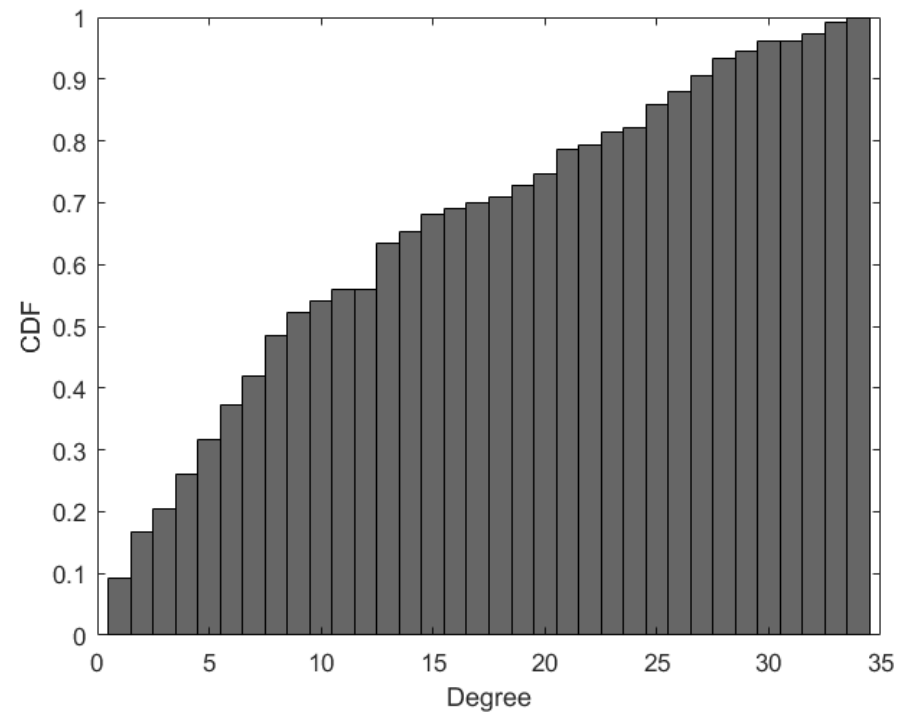

Fig. 6. Cumulative distribution function for the degree of the graph constructed using 40 features.

\section{Discussion}

In literature, classical (signal-specific) features of various physiological signals were commonly used to identify patterns of physiological threat and challenge responses. However, EMG signals were not included in any relevant work aimed at identifying such physiological patterns. To show the differences between the work we propose and that in literature, we excluded Corr and Zygo EMG signals and tested our hypotheses using only classical (i.e. signalspecific) features calculated for the rest of the signals. Results of testing the 3 hypothesis were then compared to those obtained using both classical and signal-independent features computed for all signals. Using the classical features, when performing the within-individual analysis, a maximum average accuracy of $88.23 \%$ was obtained. Approximately the same accuracy can be obtained using only 7 features instead of using the whole feature set. Compared to the within-individual analysis performed above using both signal-specific and signal-independent features on all signals, a reduction of approximately $9 \%$ in accuracy took place. On the other hand, when testing $\mathrm{H} 2$ using the top $K_{i}=7$ selected features, all of the participants fell into the same cluster. Therefore, $\mathrm{H} 2$ cannot be demonstrated since all the individuals share common subset of features and the differentiation between clusters of individuals was not apparent using only the classical feature set. Finally, to test H3, we used the self-report ratings obtained at the end of task 3 to perform the prediction analysis on all the participants since all of them formed one cluster according to H2. However, the accuracy resulted from such generalization analysis was as low as $48 \%$. Based on these results as well as the the results described above in the results section (obtained using both classical and signal-independent features applied on all signals), it can be concluded that using the signalindependent features not only highlights the differences among subjects by clustering them to 3 clusters instead of only one cluster, but also provides a higher predictive power for the inference model as shown in Table 2.

\section{Conclusion}

The present study examined multimodal physiological response patterns across motivated performance tasks of varying difficulty, with the main focus on variation within and across individuals. We designed a series of active coping stressor tasks, collected the multimodal peripheral physiological activity of participants during these tasks, and performed an intensive machine learning analysis. Our results showed that there are salient physiological features which dominate the differentiation of physiological response patterns within-individuals across tasks of varying difficulty. Moreover, individual differences were observed in terms of which physiological features are most salient for differentiating physiological responding across tasks, and we identified three groups of individuals with common salient physiological features. Our experimental results using machine learning algorithms showed that the classification accuracy of challenge v. threat (i.e., the ratio of self-report stress and coping experiences) is dramatically improved by considering an individually-tailored set of physiological features compared to using an identical set of physiological features for all participants. Our future work may further enhance the understanding of temporal dynamics of physiological responding during challenge and threat using multimodal data fusion and temporal network approaches.

\section{REFERENCES}

[1] J Blascovich and WB Mendes, "Challenge and threat appraisals: The role of affective cues. inj. forgas (ed.), feeling and thinking: The role ofaffect in social cognition (pp. 59-82)," 2000.

[2] Joe Tomaka, Jim Blascovich, Robert M Kelsey, and Christopher L Leitten, "Subjective, physiological, and behavioral effects of threat and challenge appraisal.," Journal of Personality and Social Psychology, vol. 65, no. 2, pp. 248, 1993.

[3] Joe Tomaka, Jim Blascovich, Jeffrey Kibler, and John M Ernst, "Cognitive and physiological antecedents of threat and challenge appraisal.," Journal of personality and social psychology, vol. 73, no. 1, pp. 63, 1997.

[4] Jeremy P Jamieson, Wendy Berry Mendes, Erin Blackstock, and Toni Schmader, "Turning the knots in your stomach into bows: Reappraising arousal improves performance on the gre," Journal of Experimental Social Psychology, vol. 46, no. 1, pp. 208-212, 2010. 
[5] Wendy Berry Mendes, Jim Blascovich, Brenda Major, and Mark Seery, "Challenge and threat responses during downward and upward social comparisons," European Journal of Social Psychology, vol. 31, no. 5, pp. 477-497, 2001.

[6] Wendy Berry Mendes, Jim Blascovich, Brian Lickel, and Sarah Hunter, "Challenge and threat during social interactions with white and black men," Personality and Social Psychology Bulletin, vol. 28, no. 7, pp. 939-952, 2002.

[7] Jim Blascovich, Wendy Berry Mendes, Sarah B Hunter, Brian Lickel, and Neneh Kowai-Bell, "Perceiver threat in social interactions with stigmatized others.," Journal of personality and social psychology, vol. 80, no. 2, pp. 253, 2001.

[8] Wendy Berry Mendes, Heather M Gray, Rodolfo MendozaDenton, Brenda Major, and Elissa S Epel, "Why egalitarianism might be good for your health: Physiological thriving during stressful intergroup encounters," Psychological Science, vol. 18, no. 11, pp. 991-998, 2007.

[9] Wendy Berry Mendes, Jim Blascovich, Sarah B Hunter, Brian Lickel, and John T Jost, "Threatened by the unexpected: physiological responses during social interactions with expectancy-violating partners.," Journal of personality and social psychology, vol. 92, no. 4, pp. 698, 2007.

[10] Jim Blascovich and Joe Tomaka, "The biopsychosocial model of arousal regulation," Advances in experimental social psychology, vol. 28, pp. 1-51, 1996.

[11] Richard A Dienstbier, "Arousal and physiological toughness: implications for mental and physical health.," Psychological review, vol. 96 , no. 1, pp. 84, 1989.

[12] Wendy Berry Mendes, Brenda Major, Shannon McCoy, and Jim Blascovich, "How attributional ambiguity shapes physiological and emotional responses to social rejection and acceptance.," Journal of personality and social psychology, vol. 94, no. 2, pp. 278, 2008.

[13] Jeremy P Jamieson, Matthew K Nock, and Wendy Berry Mendes, "Mind over matter: reappraising arousal improves cardiovascular and cognitive responses to stress.," Journal of Experimental Psychology: General, vol. 141, no. 3, pp. 417, 2012.

[14] Karen S Quigley, Lisa Feldman Barrett, and Suzanne Weinstein, "Cardiovascular patterns associated with threat and challenge appraisals: A within-subjects analysis," Psychophysiology, vol. 39, no. 3, pp. 292-302, 2002.

[15] Brenda Major, Wendy Berry Mendes, and John F Dovidio, "Intergroup relations and health disparities: A social psychological perspective.," Health Psychology, vol. 32, no. 5, pp. 514, 2013.

[16] Dongrui Wu and Thomas D Parsons, "Inductive transfer learning for handling individual differences in affective computing," in $A f-$ fective Computing and Intelligent Interaction, pp. 142-151. Springer, 2011.

[17] Lachezar Bozhkov, Petia Georgieva, Isabel Santos, Ana Pereira, and Carlos Silva, "Eeg-based subject independent affective computing models," Procedia Computer Science, vol. 53, pp. 375-382, 2015.

[18] Jonghwa Kim and Elisabeth André, "Emotion recognition based on physiological changes in music listening," IEEE transactions on pattern analysis and machine intelligence, vol. 30, no. 12, pp. 20672083, 2008.

[19] Jing Chen, Bin $\mathrm{Hu}$, Yue Wang, Yongqiang Dai, Yuan Yao, and Shengjie Zhao, "A three-stage decision framework for multisubject emotion recognition using physiological signals," in Bioinformatics and Biomedicine (BIBM), 2016 IEEE International Conference on. IEEE, 2016, pp. 470-474.

[20] Lawrence S Chen, Hai Tao, Thomas S Huang, Tsutomu Miyasato, and Ryohei Nakatsu, "Emotion recognition from audiovisual information," in Multimedia Signal Processing, 1998 IEEE Second Workshop on. IEEE, 1998, pp. 83-88.

[21] M. Qu, Y. Zhang, J. G. Webster, and W. J. Tompkins, “Motion artifact from spot and band electrodes during impedance cardiography," IEEE Transactions on Biomedical Engineering, vol. 33, no. 11, pp. 1029-1036, 1986.

[22] "A GUI on Biosignal-Specific Processing Pipeline," http://www. northeastern.edu/ostadabbas/codes/, Accessed: 2017.

[23] Jiapu Pan and Willis J Tompkins, "A real-time qrs detection algorithm," IEEE transactions on biomedical engineering, , no. 3, pp. 230-236, 1985.

[24] Majdi Bsoul, Hlaing Minn, Mehrdad Nourani, Gopal Gupta, and Lakshman Tamil, "Real-time sleep quality assessment using single-lead ecg and multi-stage svm classifier," in Engineering in Medicine and Biology Society (EMBC), 2010 Annual International Conference of the IEEE. IEEE, 2010, pp. 1178-1181.

[25] Philip De Chazal, Conor Heneghan, Elaine Sheridan, Richard Reilly, Philip Nolan, and Mark O'Malley, "Automated processing of the single-lead electrocardiogram for the detection of obstructive sleep apnoea," IEEE Transactions on Biomedical Engineering, vol. 50, no. 6, pp. 686-696, 2003.

[26] Kyung Hwan Kim, Seok Won Bang, and Sang Ryong Kim, "Emotion recognition system using short-term monitoring of physiological signals," Medical and biological engineering and computing, vol. 42, no. 3, pp. 419-427, 2004.

[27] Joey Benedek and R Hazlett, "Incorporating facial emg emotion measures as feedback in the software design process," Proc. Human Computer Interaction Consortium, 2005.

[28] Dennis Tkach, He Huang, and Todd A Kuiken, "Study of stability of time-domain features for electromyographic pattern recognition," Journal of neuroengineering and rehabilitation, vol. 7, no. 1, pp. 21, 2010.

[29] Simon S. Young, Computerized Data Acquisition and Analysis for the Life Sciences: A Hands-on Guide, Cambridge University Press, 2001

[30] Michael T Allen, Jochen Fahrenberg, Robert M Kelsey, William R Lovallo, Lorenz JP Doornen, et al., "Methodological guidelines for impedance cardiography," Psychophysiology, vol. 27, no. 1, pp. 1-23, 1990.

[31] Mahesh S. Chavan, R. A. Agarwala, and M. D. Uplane, "Digital elliptic filter application for noise reduction in ecg signal," 4 th WSEAS International Conference on ELECTRONICS, CONTROL and SIGNAL PROCESSING, pp. 58-63, 2005.

[32] Figner and Murphy, "Using skin conductance in judgment and decision making research," in A handbook of process tracing methods for decision research. 2011, New York, NY: Psychology Press.

[33] CJ De Luca, LD Gilmore, M Kuznetsov, and SH. Roy, "Filtering the surface emg signal: Movement artifact and baseline noise contamination.," Journal of Biomechanics, vol. 43, no. 8, pp. 15731579,2010

[34] "Knowledge Base Impedance Cardiography," https://support. mindwaretech.com/article-categories/imp/, Accessed: 2017.

[35] Peter Welch, "The use of fast fourier transform for the estimation of power spectra: a method based on time averaging over short, modified periodograms," IEEE Transactions on audio and electroacoustics, vol. 15, no. 2, pp. 70-73, 1967.

[36] Jerritta Selvaraj, Murugappan Murugappan, Khairunizam Wan, and Sazali Yaacob, "Classification of emotional states from electrocardiogram signals: a non-linear approach based on hurst," Biomedical engineering online, vol. 12, no. 1, pp. 44, 2013.

[37] AW Goertz, "Left ventricular ejection time and end-systolic pressure revisited," Anesthesia \& Analgesia, vol. 81, no. 4, pp. 890891, 1995.

[38] AM Maceira, SK Prasad, M Khan, and DJ Pennell, “Normalized left ventricular systolic and diastolic function by steady state free precession cardiovascular magnetic resonance," Journal of Cardiovascular Magnetic Resonance, vol. 8, no. 3, pp. 417-426, 2006.

[39] Robert M Kelsey and William Guethlein, "An evaluation of the ensemble averaged impedance cardiogram," Psychophysiology, vol. 27, no. 1, pp. 24-33, 1990.

[40] Yvan Saeys, Iñaki Inza, and Pedro Larrañaga, "A review of feature selection techniques in bioinformatics," bioinformatics, vol. 23, no. 19, pp. 2507-2517, 2007.

[41] Paul Jaccard, "The distribution of the flora in the alpine zone.," New phytologist, vol. 11, no. 2, pp. 37-50, 1912.

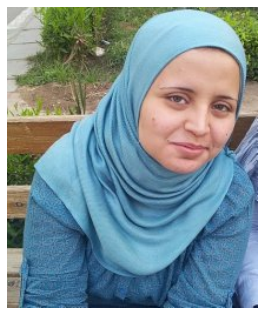

Aya Khalaf received the B.Sc. and M.Sc. degrees from the Systems and Biomedical Engineering Department, Cairo University, in 2012 and 2015 respectively. She worked as a teaching and research assistant in Cairo University since 2012. In 2015, she started her Ph.D. at Electrical and Computer Engineering Department, University of Pittsburgh. Her research interests include brain computer interfaces, computational neuroscience, and machine learning. 

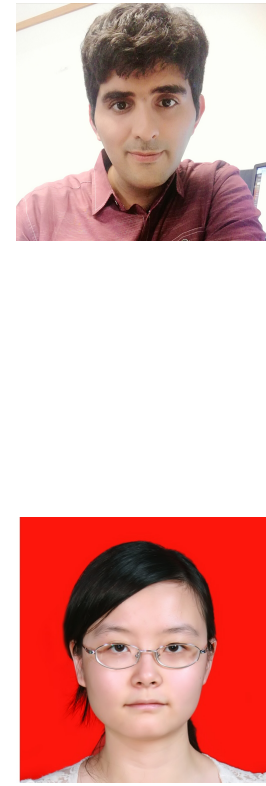

Miaolin Fan received B.Sc. degree in Applied Psychology Department, Central China Normal University, in 2012. She is currently in Ph.D. program of Mechanical and Industrial Engineering Department in Northeastern University. Her research interests include machine learning applications of neuro-imaging data mining and dynamical systems.

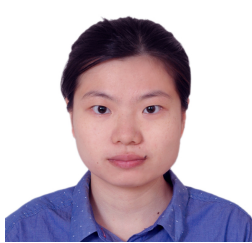

Yu Yin received B.Sc. degree in Electrical and Information Engineering Department, Wuhan University of Technology, China, in 2016. She is currently an M.Sc. student in the Electrical and Computer Engineering Department of Northeastern University. She is also a member of the Augmented Cognition Lab (ACLab). Her research interests include bio-signal processing, computer vision, and machine learning.

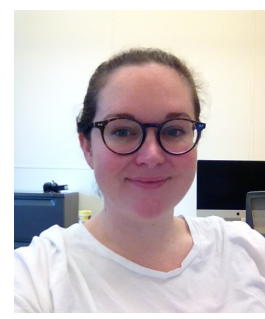

Jolie B. Wormwood received B.Sc. degrees in Mathematics and Psychology from Ithaca College. She received a M.Sc. degree and Ph.D. in Social Psychology from Northeastern University. She completed a postdoctoral research assistantship in the Interdisciplinary Affective Science Laboratory at Northeastern University, where she is currently an associate research scientist. She is interested in the role of the body in affect, decision making, and risk perception.

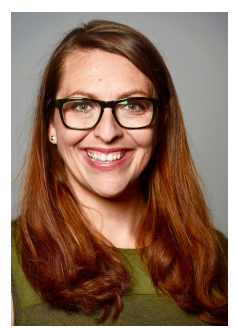

Erika H. Siegel is a postdoctoral research fellow in Health Psychology at the University of California, San Francisco. She received her Ph.D. in psychology from Northeastern University in 2015. Her work explores how affective feelings and bodily sensations help construct our experience of the world.

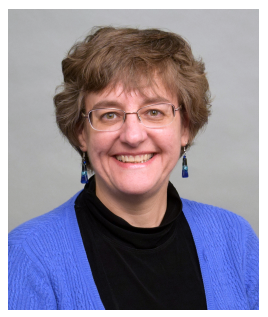

Karen Quigley received a Bachelors degree in Zoology, a Masters degree in Psychobiology, and a Ph.D. in Psychobiology from The Ohio State University before completing a postdoctoral fellowship at Columbia University. She is currently a Research Associate Professor and co-director of the Interdisciplinary Affective Science Laboratory in the Psychology Department at Northeastern University, as well as a Research Physiologist for the Center for Healthcare Organization \& Implementation Research (CHOIR) at the Edith Nourse Rogers Memorial VA Hospital, and Corresponding Director of the CHOIR Post-doctoral Research Program. Her research focuses on how humans utilize information from the body in creating emotional and affective states, individual differences in emotional reactivity, and how emotional states influence behavior, cognitions, and health.

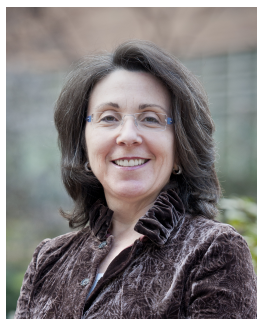

Lisa Feldman Barrett received a bachelors degree in Psychology from the University of Toronto, and a PhD in Psychology from the University of Waterloo. She is a University Distinguished Professor of Psychology and Director of the Interdisciplinary Affective Science Laboratory in the Psychology Department at Northeastern University. She also holds appointments as a Research Scientist in the Department of Psychiatry at Massachusetts General Hospital (MGH), as a Neuroscientist in the Department of Radiology at MGH, and as a Lecturer in Psychiatry at Harvard Medical School. She received a National Institutes of Health Director's Pioneer Award for her groundbreaking research on emotion in the brain, and is an elected member of the Royal Society of Canada. Her research examines emotion from both psychological and neuroscience perspectives, developing a general framework for understanding how the brain creates the mind.

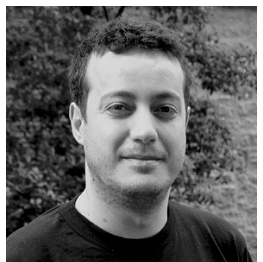

Murat Akcakaya received the B.Sc. in Electrical and Electronics Engineering Department of Middle East Technical University, Ankara, Turkey, in 2005, and the M.Sc. and the Ph.D. degree in Electrical Engineering from Washington University in St. Louis, MO, in May and December 2010, respectively. He is currently an Assistant Professor in the Electrical and Computer Engineering Department of the University of Pittsburgh. His research interests are in the area of statistical signal processing and machine learning with applications in non-invasive brain computer interface design.

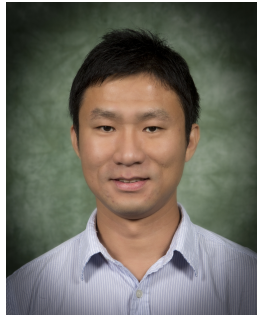

Chun-An Chou received both B.Sc. and M.Sc. degrees from National Taiwan University, M.Sc.degree in Operations Research from Columbia University in 2007, and Ph.D. degree in Industrial and Systems Engineering at Rutgers University in 2011. He is currently an Assistant Professor in the Department of Mechanical and Industrial Engineering at Northeastern University. He also held the position in the Department of Systems Science and Industrial Engineering at the State University of New York at Binghamton from 2012 to 2016. His research interests include applied large-scale optimization, machine learning, and data analytics for decision support systems in, but not limited to, healthcare and computational neuroscience. 


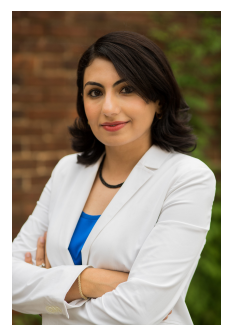

Sarah Ostadabbas received her B.Sc. in both Electrical and Biomedical Engineering from Amirkabir University of Technology, Tehran, Iran in 2005 and an M.Sc. degree in Control Engineering from Sharif University of Technology, Tehran, Iran in 2007. In 2014, she received her Ph.D. in Electrical Engineering from the University of Texas at Dallas. She is currently an Assistant Professor in the Electrical and Computer Engineering Department of Northeastern University, where she direct the Augmented Cognition Lab (ACLab) with the goal of enhancing human informationprocessing capabilities through the design of adaptive interfaces via physical, physiological, and cognitive state estimation. Her research focus is at the intersection of computer vision and machine learning with the multidisciplinary elements from behavioral sciences. 